
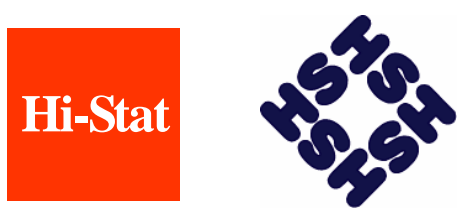

Discussion Paper Series

No.69

\title{
A Test of Serial Independence of Deviations \\ from Cointegrating Relations
}

Hiroaki Chigira

January 2005

Hitotsubashi University Research Unit for Statistical Analysis in Social Sciences

A 21st-Century COE Program

Institute of Economic Research

Hitotsubashi University

Kunitachi, Tokyo, 186-8603 Japan http://hi-stat.ier.hit-u.ac.jp/ 


\section{A TEST OF SERIAL INDEPENDENCE OF DEVIATIONS FROM COINTEGRATING RELATIONS}

HIROAKI CHIGIRA 


\section{INTRODUCTION}

The problem of cointegration, i.e. situations in time-series regression analysis where deviations from a long-run relationship follow a stationary process, has attracted much research interest in recent years. Cointegration techniques aiming to investigate for the presence of cointegrating vectors and to estimate their values have come to be widely used by econometricians. However, researchers applying cointegration methods need to pay attention not only to the cointegration relationship itself but in some cases also to the deviations from the relationship. That is to say, in some empirical applications of cointegration methods, the serial independence of the errors needs to be examined. For example, when examining the unbiasedness hypothesis in foreign exchange markets, the cointegrating regression might be specified as follows:

$$
s_{t}=\beta_{0}+\beta_{1} f_{t-1}+u_{t}
$$

where $s_{t}$ and $f_{t}$ are the natural logarithms of the spot rate and the forward rate, respectively. For the unbiasedness hypothesis to hold, $\beta_{0}$ must be equal to zero and $\beta_{1}$ equal to one. In addition, $u_{t}$ must be serially uncorrelated. The intuition underlying these requirements, in the words of Brenner and $\operatorname{Kroner}(1995,33)$ is that 'if all relevant information is immediately impounded into asset prices, then on average, the forward rate should equal the realized spot rate, and there should be no information left in the residuals to help predict future spot rates'. However, as Zivot (1998) notes, many practitioners fail to address the question of serial correlation.

In this paper, using the framework of Johansen $(1988,1991)$, we develop a method of testing whether deviations from a cointegration relationship are serially independent. The proposed test statistic is easy to calculate and asymptotically $\chi^{2}$-distributed. In order to evaluate the test, we provide Monte Carlo comparisons of the proposed test statistic and the test suggested by Kellard, Newbold, and Rayner (2001) for the size distortions and the power of the tests. The latter method is performed in two stages. In the first stage, the residual $u_{t}$ is estimated resorting to the cointegration methodology suggested by Johansen $(1988,1991)$. In the second stage, Schwartz's Bayesian information criterion ( $\mathrm{SBIC})$ is used to fit $\operatorname{ARMA}(p, q)$ models to the estimated residuals. If $\operatorname{ARMA}(0,0)$ is 
selected by SBIC, then it can then be concluded that $u_{t}$ is white noise. The Monte Carlo study indicates that in terms of power, the proposed test is superior. An empirical example using exchange rates illustrates the testing procedure and arrives at a result that is consistent with the experiment.

The next section introduces the proposed test of serial independence in detail. We formulate the test, derive the testable condition and the test statistic, and establish the limiting distribution of the test statistic. Section 3 presents the Monte Carlo results comparing our test to the method of Kellard, Newbold, and Rayner (2001). Section 4 contains a small empirical application of the test, and Section 5 concludes.

\section{TESTING FOR SERIAL INDEPENDENCE}

Consider an $m$-vector process for the cointegrating relationship generated by Johansen's vector error correction (VEC) model,

$$
\Delta x_{t}=\mu+\alpha \beta^{\prime} x_{t-1}+\sum_{j=1}^{p-1} \Gamma_{j} \Delta x_{t-j}+\Theta D_{t}+\varepsilon_{t},
$$

where $x_{t}$ is an $(m \times 1)$ vector of variables integrated of order one, $\mu$ is a constant vector, $\Gamma_{i}$ is an $(m \times m)$ matrix, $\Delta$ is the first difference operator and $\varepsilon_{t}$ is distributed $\operatorname{IIN}\left(0, \Sigma_{\varepsilon \varepsilon}\right)$. The deterministic terms $D_{t}$ can contain regressors that we consider non-stochastic such as a time trend or seasonal dummies. Supposing the system (2) is cointegrated with rank $r$ and $0<r<m, \alpha$ is a full rank $(m \times r)$ matrix and $\beta$ is a full rank $(m \times r)$ matrix consisting of $r$ cointegrating vectors such that $u_{t}=\beta^{\prime} x_{t}$ is stationary.

Our interest is in whether deviations from cointegration relations are serially uncorrelated, i.e. whether $u_{t}$ is serially uncorrelated. Obviously, whether errors from cointegration relationships are serially uncorrelated is independent of the deterministic terms $\mu$ and $D_{t}$; in what follows, we therefore drop these terms for convenience. We state the following proposition, giving the testable condition for the null hypothesis of serial independence of the deviations:

Proposition 1. Let $x_{t}$ be generated by (2) with cointegration rank $0<r<m$. Then, 
$\beta^{\prime} x_{t}$ is serially uncorrelated if and only if

$$
\beta^{\prime}\left[\begin{array}{llll}
\alpha & \Gamma_{1} & \cdots & \Gamma_{2}
\end{array}\right]=\left[\begin{array}{llll}
-I & 0 & \cdots & 0
\end{array}\right]
$$

Proof: We solve (2) for $\beta^{\prime} x_{t}$. Multiplying equation (2) by $\beta^{\prime}$ we obtain

$$
\beta^{\prime} \Delta x_{t}=\beta^{\prime} \alpha \beta^{\prime} x_{t-1}+\sum_{j=1}^{p-1} \beta^{\prime} \Gamma_{j} \Delta x_{t-j}+\beta^{\prime} \varepsilon_{t} .
$$

Therefore

$$
\left(I-\left(I+\beta^{\prime} \alpha\right) L\right) \beta^{\prime} x_{t}=\sum_{j=1}^{p-1} \beta^{\prime} \Gamma_{j} \Delta x_{t-j}+\beta^{\prime} \varepsilon_{t},
$$

where $L$ is the lag operator.

Thus, we have

$$
\beta^{\prime} x_{t}=\left(I-\left(I+\beta^{\prime} \alpha\right) L\right)^{-1}\left[\sum_{j=1}^{p-1} \beta^{\prime} \Gamma_{j} C(L) L^{j}+\beta^{\prime}\right] \varepsilon_{t}
$$

where $C(L)$ is the lag polynomial such that $\Delta x_{t}=C(L) \varepsilon_{t}$. Because $\Delta x_{t}$ is stationary by assumption, Wold's decomposition theorem ensures the existence of such $C(L)$. Equation (4) represents the time series structure of $u_{t}=\beta^{\prime} x_{t}$ itself. Substituting (3) in (4), we obtain

$$
\beta^{\prime} x_{t}=\beta^{\prime} \varepsilon_{t} .
$$

This means that if (3) holds, then deviations from cointegration relations are serially uncorrelated.

Furthermore, equation (4) establishes that the restriction that makes all the coefficients of $\varepsilon_{i}$ for $i=1,2, \cdots$ zero is only (3).

In what follows, we take (3) as the testable condition of the null of serial independence of the forecast errors. That is, we consider the following testing problem:

$$
H_{0}: \beta^{\prime}\left[\begin{array}{llll}
\alpha & \Gamma_{1} & \cdots & \Gamma_{2}
\end{array}\right]=\left[\begin{array}{llll}
-I & 0 & \cdots & 0
\end{array}\right] \text { versus } H_{1}: \operatorname{not} H_{0} .
$$

For notational convenience we denote $\left[\begin{array}{lllll}\alpha & \Gamma_{1} & \cdots & \Gamma_{2}\end{array}\right]$ and $\left[\begin{array}{llll}-I & 0 & \cdots & 0\end{array}\right]$ as $\theta$ and $I_{0}$, respectively. 
The Wald test is exploited to handle problem (5). Specifically,

$$
W=T\left\{\operatorname{vec}\left(\hat{\beta}^{\prime} \hat{\theta}\right)-\operatorname{vec}\left(I_{0}\right)\right\}^{\prime}(\hat{\Sigma})^{-1}\left\{\operatorname{vec}\left(\hat{\beta}^{\prime} \hat{\theta}\right)-\operatorname{vec}\left(I_{0}\right)\right\}
$$

where $\left.\Sigma=\beta^{\prime} \Omega \beta \otimes \Sigma_{X X}^{-1}, \Sigma_{X X}=\operatorname{Var}\left[\left(\beta^{\prime} x_{t-1}\right) ; \Delta x_{t-1} ; \cdots ; \Delta x_{t-p+1}\right)\right]$, and $\operatorname{vec}(\cdot)$ is the row-stacking operator. We do not apply the likelihood ratio test and the Lagrange multiplier test because they have the practical shortcoming that estimating (2) under the restriction (3) is difficult.

Next, we derive the limiting distribution of (6). Because the testable condition is described by the coefficient of $(2)$, we use the results of Johansen $(1998,1991)$ to establish the limiting distribution. As a result, we can easily show that (6) has a standard distribution asymptotically.

\section{Proposition 2.}

$$
W \stackrel{d}{\rightarrow} \chi_{r(r+(p-1) m)}^{2}
$$

Proof: Johansen's (1995) Theorem 13.5 states

$$
\sqrt{T}\{\operatorname{vec}(\hat{\theta})-\operatorname{vec}(\theta)\} \stackrel{d}{\rightarrow} N\left(0, \Omega \otimes \Sigma_{X X}^{-1}\right) .
$$

Hence, we obtain

$$
\begin{aligned}
\sqrt{T}\left\{\operatorname{vec}\left(\hat{\beta}^{\prime} \hat{\theta}\right)-\operatorname{vec}\left(\beta^{\prime} \theta\right)\right\} & =\sqrt{T} \operatorname{vec}\left(\beta^{\prime} \hat{\theta}\right)-\sqrt{T} \operatorname{vec}\left(\beta^{\prime} \theta\right)+\sqrt{T}\left(\operatorname{vec}\left(\hat{\beta}^{\prime} \hat{\theta}\right)-\operatorname{vec}\left(\beta^{\prime} \hat{\theta}\right)\right) \\
& \stackrel{d}{\rightarrow} N\left(\beta^{\prime} \Omega \beta \otimes \Sigma_{X X}^{-1}\right)+0 .
\end{aligned}
$$

We derive the desired result by using (7).

\section{MONTE CARLO SIMULATION}

In this section, we conduct a Monte Carlo simulation to investigate the finite-sample properties of the test statistic. The numerical performance of (6) is compared with that 
of the alternative method of testing serial independence proposed by Kellard, Newbold, and Rayner (2001).

In our experiment, the following data generating process is employed:

$$
\Delta x_{t}=\left[\begin{array}{l}
0.75 \\
-0.5
\end{array}\right]\left[\begin{array}{ll}
-1.0 & 0.5
\end{array}\right] x_{t-1}+\Gamma_{1} \Delta x_{t-1}+\varepsilon_{t},
$$

where $\varepsilon_{t}$ is i.i.d. $N\left(0, I_{2}\right)$. To test the null hypothesis of serial independence, we let

$$
\Gamma_{1}=\left[\begin{array}{cc}
0.35 & -0.35 \\
0.7 & -0.7
\end{array}\right]
$$

Under the alternative, we let

$$
\Gamma_{1}=\left[\begin{array}{cc}
0.35 & -0.35 \\
0.7 & -0.7
\end{array}\right]+\delta\left[\begin{array}{cc}
0 & 0.05 \\
-0.05 & 0
\end{array}\right]
$$

where $\delta \in\{1,2,3\}$. The sample sizes are $T=100,200,400$, and the nominal size of our test is $5 \%$.

Because model selection by SBIC used in the method proposed by Kellard, Newbold, and Rayner (2001) is not a test, the performance of that method and the test proposed here cannot be compared directly. Therefore, in order to evaluate the performance of the method used by Kellard, Newbold, and Rayner (2001), we count the number of times SBIC does not fit $\operatorname{ARMA}(0,0)$ to $\hat{\beta}^{\prime} x_{t}$, where $\hat{\beta}^{\prime}$ is the maximum likelihood estimator, based on 10,000 replications. Comparing this count with the rejection frequency of the test is an appropriate way in which the two methods can be compared.

Table 1 shows the results of the experiment. In Table 1, our test is denoted by $W$ and the method used by Kellard, Newbold, and Rayner (2001) is denoted by SBIC.

Table 1

\begin{tabular}{c|c|c}
\multicolumn{2}{c}{ size } \\
\hline \hline & $W$ & SBIC \\
\hline$T=100$ & 6.7 & 10.6 \\
$T=200$ & 6.4 & 6.6 \\
$T=400$ & 5.3 & 4.1 \\
\hline
\end{tabular}




\begin{tabular}{|c|c|c|c|c|c|c|}
\hline & \multicolumn{2}{|c|}{$\operatorname{power}(\delta=1)$} & \multicolumn{2}{|c|}{ power $(\delta=2)$} & \multicolumn{2}{|c|}{ power $(\delta=3)$} \\
\hline & $W$ & SBIC & $W$ & SBIC & $W$ & SBIC \\
\hline$T=100$ & 16.0 & 12.9 & 44.0 & 20.7 & 76.9 & 37.7 \\
\hline$T=200$ & 23.8 & 9.5 & 72.2 & 24.6 & 97.0 & 58.8 \\
\hline$T=400$ & 42.5 & 8.7 & 96.3 & 43.9 & 100.0 & 90.0 \\
\hline
\end{tabular}

The results of the experiment suggest that the size distortions of our test are moderate. When $T=400$, our test yields an accurate size. The power property of our test contrasts with the corresponding part of SBIC model selection. Under the alternative, the proportion of correct selections by SBIC, i.e., the proportion of the number of cases when SBIC does not select $\operatorname{ARMA}(0,0)$, is considerably smaller than the empirical power of our test. For example, when $\delta=2$ and $T=200$, the empirical power of our test is $72.2 \%$, while that of SBIC model selection is only $24.6 \%$. In addition, while the test's power tends to increase with $T$ and $\delta$, the proportion of correct inferences by SBIC actually decreases with $T$ when $\delta=1$. Seen in this light, our test performs considerably better than SBIC model selection.

\section{AN EMPIRICAL EXAMPLE}

In this section, we provide an empirical example to demonstrate the proposed test. As mentioned in the introduction, the unbiasedness hypothesis with regard to foreign exchange markets requires the error from the cointegration relationship to be white noise. Specifically, it is required that $u_{t}$ in equation (1) is serially independent. Equation (1) is reproduced here for convenience:

$$
s_{t}=\beta_{0}+\beta_{1} f_{t-1}+u_{t}
$$

The analysis consists of two steps. First, we use the proposed test to examine for the

presence of serial correlation in the error term $u_{t}$. Second, we also examine whether $\beta_{0}$ is equal to zero and $\beta_{1}$ is equal to one. 
We consider a bivariate VEC model,

$$
\Delta y_{t}=\mu+\alpha \beta^{\prime} y_{t-1}+\sum_{j=1}^{p-1} \Gamma_{j} \Delta y_{t-j}+\varepsilon_{t},
$$

with $y_{t}=\left[s_{t}, f_{t-1}\right]^{\prime}$, where $s_{t}$ and $f_{t}$ are the natural logarithms of the dollar-yen spot rate and the one month dollar-yen forward rate, respectively. Monthly data for the dollar-yen spot rate and the one month forward rate were taken from DataStream. The sample period for which monthly data are available is 1984:6 and to 2004:6, giving a sample size of $T=241$. $\beta^{\prime} y_{t-1}$ in (8) represents cointegration relationship (1) with an appropriate specification of the deterministic term, $\mu$.

Applying the Augmented Dickey-Fuller test to determine the degree of integration of the variables, it appears from (A) in Table 2 that $s_{t}$ and $f_{t}$ have a unit root. Next, we apply Johansen's procedure to test the cointegration rank of the system. We use SBIC to determine $p$ in equation (8) (see (B) in Table 2). (D) shows the results of the test for cointegration with specification (C). We conclude that the rank of cointegration $r$ is one because the null hypothesis of no cointegration at the $1 \%$ significance level is rejected while the null of $r \leq 1$ is not rejected at the $1 \%$ significance level. 
Table 2

(A) Test for unit root of exchange rates

\begin{tabular}{ll}
\hline & ADF test \\
\hline spot & -1.532 \\
forward & -1.374
\end{tabular}

(B) Estimated lag length of VAR: 4

(C) Estimated equation: $\Delta x_{t}=\alpha \beta^{+\prime} x_{t-1}^{+}+\sum_{j=1}^{3} \Gamma_{j} \Delta x_{t-j}+\varepsilon_{t}$, where $x_{t-1}^{+}=\left[1, x_{t-1}^{\prime}\right]^{\prime}, \beta^{+}=\left[\rho_{0}, \beta^{\prime}\right]^{\prime}$.

(D) Test for the cointegration rank

\begin{tabular}{lll}
\hline$H_{0}$ & $r=0$ & $r \leq 1$ \\
\hline trace & $25.190^{a}$ & $11.893^{b}$
\end{tabular}

We use the critical values provided by Osterwald-Lenum (1992).

${ }^{a}$ indicates rejection of the hypothesis at the $1 \%$ critical level.

${ }^{b}$ indicates rejection of the hypothesis at the $5 \%$ critical level.

(E) Standardized cointegrating vector $\hat{\beta}^{\prime}$

$$
\begin{array}{lcc}
0.008 & 1.000 \quad-1.002 \\
\left(s_{t}=0.008+1.002 f_{t-1}+u_{t}, \quad u_{t} \sim I(0)\right)
\end{array}
$$

(F) Test statistic for the null of $\alpha^{\prime} \rho_{0}=0: 0.035$

(G) Test statistic for the null of $\beta_{1}=-\beta_{2}: 0.067$

(H) Selected model for $u_{t}$ by SBIC: $\operatorname{ARMA}(0,0)$

(I) Test statistics for $\beta^{\prime} \theta=I_{0}: 19.045^{a}$

Note: ${ }^{a}$ indicates rejection of the hypothesis at the $1 \%$ critical level.

$(\mathrm{F})$ and $(\mathrm{G})$ report likelihood ratio test statistics based on Johansen's (1995) Theorem 11.3 and Theorem 7.2, respectively. These statistics are asymptotically $\chi^{2}$-distributed. The degrees of freedom depend on $m$, the dimension of the system, $r$, the cointegration rank, and the null hypothesis itself. In this empirical example, the degree of freedom is one. (F) and $(\mathrm{G})$ show that the stationary combination $s_{t}=f_{t-1}+u_{t}$ holds. The next test is to check for serial correlation in $u_{t}$. As shown in $(\mathrm{H}), \operatorname{SBIC}$ selects $\operatorname{ARMA}(0,0)$ for the estimated residual $u_{t}$, i.e., $u_{t}$ is found to be uncorrelated. Therefore, the unbiasedness 
hypothesis is accepted. On the other hand, our test rejects the null of independence at the $1 \%$ significance level as shown in (I). The result appears to be consistent with our Monte Carlo experiment. Section 3 indicated that while our test had reasonable empirical power, SBIC tended to select $\operatorname{ARMA}(0,0)$ or failed to detect dependence of $u_{t}$ in the Monte Carlo experiment. Our test suggests that there remains some information in the residuals that may help forecast the future spot rate. While the method proposed by Kellard, Newbold, and Rayner (2001) would have led one to accept the unbiasedness hypothesis, the proposed test suggests that $u_{t}$ is in fact serially correlated and the unbiasedness hypothesis should be rejected.

\section{CONCLUSION}

In this paper, we proposed a method to test the null of serial independence of a deviation from a cointegration relation. Whether deviations are serially independent is an important issue in the study of economics and finance, and the test proposed here offers various benefits: The test statistic is easy to calculate and is asymptotically $\chi^{2}$ distributed. Moreover, our test performs well in the mean in that its size distortion is moderate and it showed reasonably high power in the Monte Carlo experiment. The empirical example examining the unbiasedness hypothesis with regard to foreign exchange rates yielded results corroborating that our test has high power. 


\section{References}

Brenner, R.J. and K.F. Kroner (1995): “Arbitrage, Cointegration, and Testing the Unbiasedness Hypothesis in Financial Markets," Journal of Financial and Quantitative Analysis, 30, 23-42.

Johansen, S. (1988): "Statistical Analysis of Cointegration Vectors," Journal of Economic Dynamics and Control, 12, 231-254.

Johansen, S. (1991): "Estimation and Hypothesis Testing of Cointegration Vectors in Gaussian Vector Autoregressive Models," Econometrica, 59, 1551-1580.

Johansen, S. (1995): Likelihood-based Inference in Cointegrated Vector Autoregressive Models, Oxford University Press, Oxford.

Kellard, N., Newbold, P. and T. Rayner (2001): "Evaluating currency market efficiency: are cointegration tests appropriate? ," Applied Financial Economics, 11, 681-691.

Osterwald-Lenum, M. (1992): "A Note with Quantiles of the Asymptotic Distribution of the Maximum Likelihood Cointegration Rank Test Statistics," Oxford Bulletin of Economics and Statistics, 54, 461-472.

Zivot, E. (1998): "Cointegration and forward and spot exchange rate regressions," University of Washington, http://econwpa.wustl.edu/eps/em/papers/9812/9812001.pdf 